\title{
An exploration of the black hole entropy via the Weyl tensor
}

\author{
Nan $\mathbf{L i}^{1, a}$, Xiao-Long $\mathbf{L i}^{2, b}$, Shu-Peng Song ${ }^{3, c}$ \\ ${ }^{1}$ Department of Physics, College of Sciences, Northeastern University, Shenyang 110819, China \\ ${ }^{2}$ Department of Astronomy, Beijing Normal University, Beijing 100875, China \\ ${ }^{3}$ Department of Physics, Beijing Normal University, Beijing 100875, China
}

Received: 2 November 2015 / Accepted: 19 February 2016 / Published online: 1 March 2016

(c) The Author(s) 2016. This article is published with open access at Springerlink.com

\begin{abstract}
The role of the Weyl tensor $C_{\mu \nu \lambda \rho}$ in black hole thermodynamics is explored by looking at the relation between the scalar invariant $C_{\mu \nu \lambda \rho} C^{\mu \nu \lambda \rho}$ and the entropy of $n$-dimensional static black holes. It is found that this invariant can be identified as the entropy density of the gravitational fields for classical 5-dimensional black holes. We calculate the proper volume integrals of $C_{\mu \nu \lambda \rho} C^{\mu \nu \lambda \rho}$ for the Schwarzschild and Schwarzschild-anti-de Sitter black holes and show that these integrals correctly lead to the Bekenstein-Hawking entropy formulas, only up to some coefficients.
\end{abstract}

\section{Introduction}

From a mathematical point of view, the curvature of a manifold is measured by the 4th-order Riemann tensor $R_{\mu \nu \lambda \rho}$. In an $n$-dimensional space-time, the Riemann tensor can be decomposed into the Ricci and Weyl sectors,

$$
\begin{aligned}
R_{\mu \nu \lambda \rho}= & \frac{2}{n-2}\left(g_{\mu[\lambda} R_{\rho] \nu}-g_{\nu[\lambda} R_{\rho] \mu}\right) \\
& -\frac{2}{(n-1)(n-2)} g_{\mu[\rho} g_{\lambda] \nu} R+C_{\mu \nu \lambda \rho},
\end{aligned}
$$

with $R_{\mu \nu}$ being the 2 nd-order Ricci tensor, $R$ the Ricci scalar, and $C_{\mu \nu \lambda \rho}$ the 4th-order Weyl tensor. However, in the theory of general relativity, the Einstein equations only associate the 2nd-order Ricci tensor with the energy-momentum tensor. Therefore, a natural question is why the information encoded in the Weyl tensor is absent in general relativity. In other words, what is the role of the Weyl tensor in the theory of gravitation?

\footnotetext{
a e-mail: linan@mail.neu.edu.cn

b e-mail: lixiaolong@mail.bnu.edu.cn

c e-mail: songsp@mail.bnu.edu.cn
}

Since the Weyl tensor is locally independent of the energymomentum tensor, it may be viewed as a purely geometrical description of the curvature of space-time. Thus, the Weyl tensor is linked not to the dynamical, but possibly to the thermodynamical aspects of gravitational fields. One of the explorations in this direction is the "Weyl curvature conjecture", or the "Penrose conjecture" [1].

The Weyl tensor is traceless and has the same symmetries as the Riemann tensor, and the metric of a space-time is conformally flat, if its Weyl tensor vanishes. During the cosmological evolution, our universe evolves from an almost homogeneous and isotropic space-time (conformally flat with vanishing Weyl tensor) to an ensemble of randomly distributed black holes (not conformally flat with non-vanishing Weyl tensor). As a consequence, the Weyl tensor seems to grow monotonically in the universe, and this reminds us of the second law of thermodynamics. Therefore, Penrose conjectured that some scalar invariant of the Weyl tensor (e.g. $C_{\mu \nu \lambda \rho} C^{\mu \nu \lambda \rho}$ ) is a monotonically growing function of time and is thus somehow related to the gravitational entropy in the universe. Some previous studies of the Penrose conjecture in the theory of gravitation can be found in Refs. [2-12] and in cosmology in Refs. [13-21].

Unfortunately, despite various attempts, Penrose's idea has still not been formulated in a rigorous way. This difficulty lies in the fact that it is questionable to generally define a gravitational entropy by means of scalar invariants, not only because the entropy itself is not mathematically a scalar, but also because many scalars constructed with the Weyl tensor are not monotonically increasing and thus cannot provide an appropriate measure of the gravitational entropy. For instance, the invariant function $C_{\mu \nu \lambda \rho} C^{\mu \nu \lambda \rho} / R_{\mu \nu} R^{\mu \nu}$ suggested in Refs. [22-24], which helps to solve the initial isotropic singularity problem, is ill-defined in vacuum where $R_{\mu \nu}=0$. This early work on the Penrose conjecture [2-24] was systematically summarized and commented on in Ref. [25], where the authors also proposed a measure from the 
Bel-Robinson tensor and showed that this measure is applicable to some exact or perturbative models under certain conditions. However, to our present knowledge, the precise and definite notion of entropy and its relation to the Weyl tensor are still unclear and have to be specified. The aim of this paper is just to investigate the possible connection between them. In the following sections, we will explore the possibilities to calculate the entropies of the Schwarzschild and Schwarzschild-anti-de Sitter black holes via the Weyl invariant, respectively.

\section{Entropy of the Schwarzschild black hole from the Weyl invariant}

Below, we choose the natural units system, with $c=\hbar=$ $k_{\mathrm{B}}=1$, but keep the gravitational constant $G$, as we will work in different dimensional space-times, in which the gravitational constants are not the same.

We first explain the Penrose conjecture in a more quantitative way. The metric of a Schwarzschild black hole reads $\mathrm{d} s^{2}=-\left(1-R_{\mathrm{S}} / r\right) \mathrm{d} t^{2}+\left(1-R_{\mathrm{S}} / r\right)^{-1} \mathrm{~d} r^{2}+r^{2} \mathrm{~d} \Omega_{2}^{2}$, with $R_{\mathrm{S}}=2 G M$ being the horizon radius. From this metric, the corresponding Weyl invariant is

$C_{\mu \nu \lambda \rho} C^{\mu \nu \lambda \rho}=\frac{12 R_{\mathrm{S}}^{2}}{r^{6}}$

Meanwhile, its Bekenstein-Hawking entropy is [26,27]

$S=\frac{A}{4 G}=\frac{4 \pi R_{\mathrm{S}}^{2}}{4 G}$

where $A=4 \pi R_{\mathrm{S}}^{2}$ is the area of its horizon. From Eqs. (2) and (3), we clearly observe that $C_{\mu \nu \lambda \rho} C^{\mu \nu \lambda \rho}$ is proportional to $S$, and this proportion naturally inspires us to wonder if there is some latent relation in between.

We see from Eq. (2) that $C_{\mu \nu \lambda \rho} C^{\mu \nu \lambda \rho}$ is a function of radial coordinate $r$. Moreover, from the functional form of $C_{\mu \nu \lambda \rho} C^{\mu \nu \lambda \rho}$, the Weyl invariant does not decrease inside the horizon, if evaluated along the world-lines of all physical observers. Consequently, we may interpret it as the entropy density of gravitational field of the Schwarzschild black hole, and its proper volume integral may thus give the BekensteinHawking entropy. This is the main idea of our exploration of the Penrose conjecture in the present paper.

In the following, we generally consider an $n$-dimensional Schwarzschild black hole with the metric [28]

$$
\mathrm{d} s^{2}=-f(r) \mathrm{d} t^{2}+f(r)^{-1} \mathrm{~d} r^{2}+r^{2} \mathrm{~d} \Omega_{n-2}^{2},
$$

where

$f(r)=1-\frac{R_{n}^{n-3}}{r^{n-3}}, \quad$ with $\quad R_{n}=\left[\frac{16 \pi G_{n} M}{(n-2) \Omega_{n-2}}\right]^{1 /(n-3)}$

Above, $R_{n}$ is the horizon radius, $G_{n}$ is the $n$-dimensional gravitational constant, and $\Omega_{n-2}=2 \pi^{(n-1) / 2} / \Gamma((n-1) / 2)$ is the solid angle of the $(n-2)$-dimensional sphere. A straightforward calculation shows

$C_{\mu \nu \lambda \rho} C^{\mu \nu \lambda \rho}=\frac{(n-2)^{2}\left[(n-2)^{2}-1\right] R_{n}^{2 n-6}}{r^{2 n-2}}$.

This contraction can also be obtained via the identity,

$C_{\mu \nu \lambda \rho} C^{\mu \nu \lambda \rho}=R_{\mu \nu \lambda \rho} R^{\mu \nu \lambda \rho}-\frac{4 R_{\mu \nu} R^{\mu \nu}}{n-2}+\frac{2 R^{2}}{(n-1)(n-2)}$,

where $R_{\mu \nu \lambda \rho} R^{\mu \nu \lambda \rho}$ is the Kretschmann invariant. The calculation in this way is much easier, since both the Ricci tensor and the Ricci scalar vanish for the Schwarzschild metric. Furthermore, the Bekenstein-Hawking entropy of the $n$-dimensional Schwarzschild black hole is [28]

$S_{n}=\frac{A_{n}}{4 G_{n}}=\frac{\Omega_{n-2} R_{n}^{n-2}}{4 G_{n}}$,

where $A_{n}=\Omega_{n-2} R_{n}^{n-2}$ is the area of the $(n-2)$-dimensional sphere at the horizon.

Now, the problem is whether the proper volume integral of $C_{\mu \nu \lambda \rho} C^{\mu \nu \lambda \rho}$ in Eq. (4) gives the entropy formula in Eq. (5). However, a deeper consideration easily invalidates this simple attempt. The dimension of the Weyl tensor, $\left[C_{\mu \nu \lambda \rho}\right]$, is +2 in the natural units system, so the dimension of $\left[C_{\mu \nu \lambda \rho} C^{\mu \nu \lambda \rho}\right]$ should be +4 . On the other hand, in an $n$-dimensional space-time, the dimension of the proper volume element $\left[\mathrm{d} V_{n-1}\right]$ is $-(n-1)$. Therefore, we cannot generally expect $C_{\mu \nu \lambda \rho} C^{\mu \nu \lambda \rho}$ as the entropy density of gravitational field in the $n$-dimensional space-time, as the entropy itself is dimensionless in the natural units system.

This dimensional analysis indicates that we are allowed to regard $C_{\mu \nu \lambda \rho} C^{\mu \nu \lambda \rho}$ as the entropy density only in a 5-dimensional space-time (not the ordinary 4-dimensional one), in which $\left[\mathrm{d} V_{4}\right]=-4$, and the proper volume integral

$\int C_{\mu \nu \lambda \rho} C^{\mu \nu \lambda \rho} \mathrm{d} V_{4}$

is dimensionless. We will show in the following that this integral does lead to the correct entropy formulas for the 5dimensional Schwarzschild and Schwarzschild-anti-de Sitter black holes, only up to some coefficients. Generally speaking, the proper volume integral of a quantity in an $n$-dimensional space-time, evaluated along an $(n-1)$ dimensional hyper-surface, strongly depends on the choice 
of space-time slicing. However, this complexity and ambiguity can be easily avoided for the Schwarzschild and Schwarzschild-anti-de Sitter black holes, as in these spherically symmetric space-times, the choice of slicing is natural, which is provided by the hyper-surfaces of constant $t$ orthogonal to the Killing vector $\partial / \partial t$.

The integral in Eq. (6) consists of three parts: the integrand $C_{\mu \nu \lambda \rho} C^{\mu \nu \lambda \rho}$, the 4-dimensional proper volume element $\mathrm{d} V_{4}$, and the domain of integration. Below, we discuss them in order.

For $C_{\mu \nu \lambda \rho} C^{\mu \nu \lambda \rho}$, the metric of the Schwarzschild black hole in 5-dimensional space-time reads

$\mathrm{d} s^{2}=-f(r) \mathrm{d} t^{2}+f(r)^{-1} \mathrm{~d} r^{2}+r^{2} \mathrm{~d} \Omega_{3}^{2}$,

where

$f(r)=1-\frac{R_{5}^{2}}{r^{2}}, \quad$ with $\quad R_{5}=\sqrt{\frac{8 G_{5} M}{3 \pi}}$.

From Eq. (4), we have the Weyl invariant,

$$
C_{\mu \nu \lambda \rho} C^{\mu \nu \lambda \rho}=\frac{72 R_{5}^{4}}{r^{8}} .
$$

For the proper volume element $\mathrm{d} V_{4}$, we have

$\mathrm{d} V_{4}=r^{3} \sqrt{\left|g_{r r}\right|} \mathrm{d} r \mathrm{~d} \Omega_{3}$.

We may first integrate the angular parts in Eq. (6),

$$
\begin{aligned}
\int C_{\mu \nu \lambda \rho} C^{\mu \nu \lambda \rho} \mathrm{d} V_{4} & =\int C_{\mu \nu \lambda \rho} C^{\mu \nu \lambda \rho} r^{3} \sqrt{\left|g_{r r}\right|} \mathrm{d} r \mathrm{~d} \Omega_{3} \\
& =\int C_{\mu \nu \lambda \rho} C^{\mu \nu \lambda \rho} \frac{2 \pi^{2} r^{3}}{\sqrt{|f(r)|}} \mathrm{d} r
\end{aligned}
$$

where $\int \mathrm{d} \Omega_{3}=\Omega_{3}=2 \pi^{2}$ is the solid angle of the 3dimensional sphere, and $\sqrt{\left|g_{r r}\right|} \mathrm{d} r=\mathrm{d} r / \sqrt{|f(r)|}$ is the proper distance element in radial direction. Here, we should state that the hyper-surfaces of constant $t$, along which the proper volume integral of $C_{\mu \nu \lambda \rho} C^{\mu \nu \lambda \rho}$ is evaluated, change their causal structures at the horizon. For $r>R_{5}$, the Killing vector $\partial / \partial t$ is time-like, so the hyper-surfaces of constant $t$ are space-like, but for $r<R_{5}$, these hyper-surfaces become time-like, as $\partial / \partial t$ is space-like. This fact makes the integral in Eq. (8) not an ordinary volume integral of a density that is performed along space-like slices in the range $r<R_{5}$.

For the domain of integration for radial coordinate $r$, the upper limit can be safely set to be infinity, but the lower limit cannot be simply taken as 0 , which diverges the integral in Eq. (8). Actually, the classical theory of general relativity is invalid at extremely small radius (about the Planck length). Therefore, we set the lower limit of $r$ to be the 5-dimensional
Planck length $l_{5}$, which can be expressed in terms of the 5dimensional gravitational constant $G_{5}$ as

$l_{5}=\sqrt[3]{G_{5}}$.

With all these preparations, substituting Eq. (7) into (8), we attain the proper volume integral of the Weyl invariant for the 5-dimensional Schwarzschild black hole,

$$
\begin{aligned}
& \int C_{\mu \nu \lambda \rho} C^{\mu \nu \lambda \rho} \mathrm{d} V_{4} \\
& =\int_{l_{5}}^{\infty} \frac{72 R_{5}^{4}}{r^{8}} \frac{2 \pi^{2} r^{3}}{\sqrt{|f(r)|}} \mathrm{d} r \\
& =144 \pi^{2} R_{5}^{4}\left(\int_{l_{5}}^{R_{5}} \frac{\mathrm{d} r}{r^{5} \sqrt{-f(r)}}+\int_{R_{5}}^{\infty} \frac{\mathrm{d} r}{r^{5} \sqrt{f(r)}}\right) \\
& =48 \pi^{2}\left\{\left[\left(\frac{R_{5}}{l_{5}}\right)^{3}+\frac{2 R_{5}}{l_{5}}\right] \sqrt{1-\left(\frac{l_{5}}{R_{5}}\right)^{2}}+2\right\} .
\end{aligned}
$$

This result seems lengthy at first glance, but in fact not. If $M \gg 1 / l_{5}$, the horizon radius of the Schwarzschild black hole $R_{5}$ is much larger than the Planck length $l_{5}$. Therefore, in the limit $R_{5} \gg l_{5}$, the above result can be significantly simplified, with the leading term being

$\int C_{\mu \nu \lambda \rho} C^{\mu \nu \lambda \rho} \mathrm{d} V_{4} \approx 48 \pi^{2}\left(\frac{R_{5}}{l_{5}}\right)^{3}$

In fact, we should mention here that the integral outside the horizon $\left(R_{5}<r<\infty\right)$ only contributes a trivial constant, as $144 \pi^{2} R_{5}^{4} \int_{R_{5}}^{\infty} \mathrm{d} r /\left(r^{5} \sqrt{f(r)}\right)=2 / 3$. This means that the result in Eq. (9) remains valid, if the integral is evaluated only inside the horizon $\left(l_{5}<r<R_{5}\right)$.

On the other hand, the Bekenstein-Hawking entropy for the 5-dimensional Schwarzschild black hole is

$S_{5}=\frac{A_{5}}{4 G_{5}}=\frac{\pi^{2}}{2}\left(\frac{R_{5}}{l_{5}}\right)^{3}$,

with $A_{5}=2 \pi^{2} R_{5}^{3}$. To this point, we eventually find that the proper volume integral of the Weyl invariant indeed leads to the correct entropy formula, only up to a coefficient,

$S_{5}=\frac{1}{96} \int C_{\mu \nu \lambda \rho} C^{\mu \nu \lambda \rho} \mathrm{d} V_{4}$

From this result, we are convinced that the interpretation of the Weyl invariant $C_{\mu \nu \lambda \rho} C^{\mu \nu \lambda \rho}$ as the entropy density for the 5-dimensional Schwarzschild black hole is reasonable. 


\section{Entropy of the Schwarzschild-anti-de Sitter black hole from the Weyl invariant}

Till now, we only discuss the simplest Schwarzschild solution, for which the Weyl tensor is identical to the Riemann tensor, $C_{\mu \nu \lambda \rho}=R_{\mu \nu \lambda \rho}$, and hence the Weyl invariant coincides with the Kretschmann invariant, so the characteristic of the Weyl tensor is not very distinct. Therefore, we further explore a more complicated black hole with non-vanishing Ricci tensor and Ricci scalar: the Schwarzschild-anti-de Sitter solution (i.e. the Schwarzschild solution with a cosmological constant $\Lambda<0$ ). In 5-dimensional space-time, its metric reads

$\mathrm{d} s^{2}=-f(r) \mathrm{d} t^{2}+f(r)^{-1} \mathrm{~d} r^{2}+r^{2} \mathrm{~d} \Omega_{3}^{2}$,

with

$f(r)=1-\frac{R_{5}^{2}}{r^{2}}-\frac{\Lambda r^{2}}{6}$.

In this case, both the Ricci tensor and the Ricci scalar are non-vanishing: $R_{\mu \nu}=\frac{2}{3} \Lambda g_{\mu \nu}$ and $R=\frac{10}{3} \Lambda$. Therefore, the difference between the Weyl and Kretschmann invariants becomes much more notable. It is straightforward to see that the Weyl invariant remains unchanged,

$C_{\mu \nu \lambda \rho} C^{\mu \nu \lambda \rho}=\frac{72 R_{5}^{4}}{r^{8}}$,

but the Kretschmann invariant now receives an additive constant from $\Lambda$,

$R_{\mu \nu \lambda \rho} R^{\mu \nu \lambda \rho}=\frac{72 R_{5}^{4}}{r^{8}}+\frac{10 \Lambda^{2}}{9}$.

These results strongly support our interpretation of the Weyl invariant, but not the Kretschmann one, as the entropy density of gravitational field. Below, we perform the parallel procedure for the proper volume integral in Eq. (8), in order to check the validity of our interpretation. ${ }^{1}$

${ }^{1}$ For completeness, we also list the relevant physical quantities for the $n$-dimensional Schwarzschild-anti-de Sitter black hole,

$$
\begin{aligned}
f(r) & =1-\frac{R_{n}^{n-3}}{r^{n-3}}-\frac{2 \Lambda r^{2}}{(n-1)(n-2)}, \\
C_{\mu \nu \lambda \rho} C^{\mu \nu \lambda \rho} & =\frac{(n-2)^{2}\left[(n-2)^{2}-1\right] R_{n}^{2 n-6}}{r^{2 n-2}}, \\
R_{\mu \nu \lambda \rho} R^{\mu \nu \lambda \rho} & =\frac{(n-2)^{2}\left[(n-2)^{2}-1\right] R_{n}^{2 n-6}}{r^{2 n-2}}+\frac{8 n \Lambda^{2}}{(n-1)(n-2)^{2}} .
\end{aligned}
$$

In a similar way, we have

$$
\begin{aligned}
& \int C_{\mu \nu \lambda \rho} C^{\mu \nu \lambda \rho} \mathrm{d} V_{4}=\int_{l_{5}}^{\infty} \frac{72 R_{5}^{4}}{r^{8}} \frac{2 \pi^{2} r^{3} \mathrm{~d} r}{\sqrt{|f(r)|}} \\
& =144 \pi^{2} R_{5}^{4}\left(\int_{l_{5}}^{R_{5}^{\prime}} \frac{\mathrm{d} r}{r^{5} \sqrt{-f(r)}}+\int_{R_{5}^{\prime}}^{\infty} \frac{\mathrm{d} r}{r^{5} \sqrt{f(r)}}\right),
\end{aligned}
$$

where

$R_{5}^{\prime}=\sqrt{\frac{3}{\Lambda}\left(1-\sqrt{1-\frac{2 \Lambda R_{5}^{2}}{3}}\right)}$

is the unique zero for $f(r)=0$ (since $\Lambda<0$ ). The results of the integrals in Eq. (13) can be expressed analytically via the elliptic functions, which depend on $R_{5}$ and $\Lambda$. However, the exact and tedious expressions are irrelevant, but only the leading term is important,

$\int C_{\mu \nu \lambda \rho} C^{\mu \nu \lambda \rho} \mathrm{d} V_{4} \approx 48 \pi^{2}\left(\frac{R_{5}}{l_{5}}\right)^{3}$.

This result is the same as that in Eq. (9), indicating that the cosmological constant does not significantly affect the integral in Eq. (13). This is not difficult to understand, as the cosmological constant contributes to the integral only at large $r$, where its effect is dominantly suppressed by the factor $1 / r^{8}$ in the Weyl invariant, so we safely arrive at the same result in Eq. (9).

Meanwhile, the Bekenstein-Hawking entropy for the 5dimensional Schwarzschild-anti-de Sitter black hole is [29]

$S_{5}^{\prime}=\frac{A_{5}^{\prime}}{4 G_{5}}=\frac{\pi^{2}}{2}\left(\frac{R_{5}^{\prime}}{l_{5}}\right)^{3}$,

with $A_{5}^{\prime}=2 \pi^{2} R_{5}^{\prime 3}$. Thus, we have

$S_{5}^{\prime}=\frac{1}{96}\left(\frac{R_{5}^{\prime}}{R_{5}}\right)^{3} \int C_{\mu \nu \lambda \rho} C^{\mu \nu \lambda \rho} \mathrm{d} V_{4}$

Hence, we again obtain the entropy formula, but this time the coefficient is $\Lambda$-dependent. From Eq. (14), for a small negative $\Lambda,\left(R_{5}^{\prime} / R_{5}\right)^{3} \rightarrow 1$; for a large negative $\Lambda,\left(R_{5}^{\prime} / R_{5}\right)^{3} \rightarrow$ $\left[6 /\left(-\Lambda R_{5}^{2}\right)\right]^{3 / 4}$. Although the coefficient varies with $\Lambda$, the proportion remains the same.

In short, for the 5-dimensional Schwarzschild-anti-de Sitter black hole, for which the Weyl tensor deviates from the Riemann tensor, $C_{\mu \nu \lambda \rho} \neq R_{\mu \nu \lambda \rho}$, we are still able to obtain the Bekenstein-Hawking entropy by integrating the Weyl invariant $C_{\mu \nu \lambda \rho} C^{\mu \nu \lambda \rho}$, as the effect of the cosmological constant is suppressed at large radius. Especially, from 
Eqs. (11) and (12), we explicitly see the difference between the Weyl invariant, $C_{\mu \nu \lambda \rho} C^{\mu \nu \lambda \rho}$, and the Kretschmann one, $R_{\mu \nu \lambda \rho} R^{\mu \nu \lambda \rho}$. We find that $C_{\mu \nu \lambda \rho} C^{\mu \nu \lambda \rho}$ is not altered in the presence of the cosmological constant, and this fact strengthens our interpretation of the Weyl invariant as the entropy density, but not the Kretschmann invariant, since it receives a constant modification $10 \Lambda^{2} / 9$, which diverges the proper volume integral at $r \rightarrow \infty$.

Here, we should also emphasize that the thermodynamics of the Schwarzschild-de Sitter black hole (i.e. the Schwarzschild solution with a positive cosmological constant) is not a well-defined issue [30-36], and we skip the corresponding discussion in the present paper.

\section{Conclusions and discussions}

Finally, we give some brief discussions of our work. The issue of entropy is one of the central problems in black hole thermodynamics [37-41], and the Penrose conjecture on the Weyl tensor is one of the possible ways to approach this issue. We try in this paper to physically confirm and mathematically formulate the Penrose conjecture. We take the Schwarzschild and Schwarzschild-anti-de Sitter black holes as examples and find that we may interpret the Weyl invariant $C_{\mu \nu \lambda \rho} C^{\mu \nu \lambda \rho}$ as their entropy densities, but this interpretation should be realized in 5-dimensional space-time as follows from the dimensional analysis. We perform the proper volume integral of $C_{\mu \nu \lambda \rho} C^{\mu \nu \lambda \rho}$ from the 5-dimensional Planck length to infinity, and we discover that this integral really results in the correct Bekenstein-Hawking entropy formulas, only up to some coefficients.

At the same time, we should also point out the limits of our work. First, our calculation only applies in 5-dimensional space-time, but not the ordinary 4-dimensional one. We may otherwise imagine that the mass of the 5-dimensional black hole is distributed on an extra dimension, and if this dimension is wrapped to an extremely small scale, we can still utilize our method to calculate the volume integral of the corresponding Weyl invariant. But in this case, we are faced with the calculation of the metric and the Weyl tensor of a black string, and this calculation is much more complicated and is thus beyond our preliminary exploration. (For the metric solution for a black string, see Refs. [42,43].) Second, we should admit that it is difficult to extend our results to more general black holes (e.g. the charged Reissner-Nordström black hole), because its geometry near the origin is quite different from that of the Schwarzschild and Schwarzschildanti-de Sitter black holes.

In summary, our work helps to understand Penrose's idea and indicates that the Weyl tensor may be related to the entropy of gravitational fields, but some difficulties are still to be overcome. This exploration leads us to investigate whether there exist equations that are parallel to the Einstein equations and quantify the thermodynamical relation between spacetime and matter. These equations are expected to relate the Weyl tensor to the thermodynamical concepts such as entropy and temperature, and we wish that our work will be conducive to the research in this direction.

Acknowledgments We are very grateful to Tian-Fu Fu, Xiao-Ran Han, and Dominik J. Schwarz for fruitful discussions. This work is supported by the Fundamental Research Funds for the Central Universities of China (No. N140504008).

Open Access This article is distributed under the terms of the Creative Commons Attribution 4.0 International License (http://creativecomm ons.org/licenses/by/4.0/), which permits unrestricted use, distribution, and reproduction in any medium, provided you give appropriate credit to the original author(s) and the source, provide a link to the Creative Commons license, and indicate if changes were made. Funded by $\mathrm{SCOAP}^{3}$.

\section{References}

1. R. Penrose, in General Relativity, an Einstein Centenary Survey, ed. by S.W. Hawking, W. Israel (Cambridge University Press, Cambridge, 1979), p. 581

2. J. Wainwright, Gen. Relativ. Gravit. 16, 657 (1984)

3. S.W. Goode, J. Wainwright, Class. Quantum Gravity 2, 99 (1984)

4. W.B. Bonnor, Class. Quantum Gravity 3, 495 (1986)

5. S.W. Goode, Class. Quantum Gravity 8, L1 (1991)

6. R.P.A.C. Newman, Proc. Roy. Soc. Lond. A 443, 473 (1993)

7. N. Pelavas, K. Lake, Phys. Rev. D 62, 044009 (2000)

8. Ø. Grøn, S. Hervik. arXiv:gr-qc/0205026

9. J.D. Barrow, S. Hervik, Class. Quantum Gravity 19, 5173 (2002)

10. Ø. Rudjord, Ø. Grøn, Phys. Scripta 77, 055901 (2008)

11. T. Maki, M. Morita, Springer Proc. Math. Stat. 60, 311 (2014)

12. R.A. Sussman, J. Larena, Class. Quantum Gravity 31, 075021 (2014)

13. V. Husain, Phys. Rev. D 38, 3314 (1988)

14. S.A. Hayward, Class. Quantum Gravity 10, L7 (1993)

15. A.V. Nesteruk, Class. Quantum Gravity 11, L15 (1994)

16. S. Barve, T.P. Singh. arXiv:gr-qc/9705060

17. W.C. Lim, H. van Elst, C. Uggla, J. Wainwright, Phys. Rev. D 69, 103507 (2004)

18. N. Li et al., Phys. Rev. D 86, 083539 (2012)

19. O.-C. Stoica, Ann. Phys. 338, 186 (2013)

20. N. Li, X.-L. Li, S.-P. Song, Eur. Phys. J. C 75, 114 (2015)

21. G. Marozzi, J.P. Uzan, O. Umeh, C. Clarkson, Gen. Relativ. Gravit. 47, 114 (2015)

22. W.B. Bonnor, Phys. Lett. A 122, 305 (1987)

23. S.W. Goode, A.A. Coley, J. Wainwright, Class. Quantum Gravity 9, 445 (1992) and the references therein

24. T. Rothman, P. Anninos, Phys. Rev. D 55, 1948 (1997)

25. T. Clifton, G.F.R. Ellis, R. Tavakol, Class. Quantum Gravity 30, 125009 (2013)

26. J.D. Bekenstein, Phys. Rev. D 7, 2333 (1973)

27. J.M. Bardeen, B. Carter, S.W. Hawking, Commun. Math. Phys. 31, 161 (1973)

28. R. Gregory, in Black Holes in Higher Dimensions, ed. by G.T. Horowitz (Cambridge University Press, Cambridge, 2012), p. 29

29. S.W. Hawking, D.N. Page, Commun. Math. Phys. 87, 577 (1983)

30. R. Bousso, S.W. Hawking, Phys. Rev. D 57, 2436 (1998)

31. Z. Stuchlík, S. Hledík, Phys. Rev. D 60, 044006 (1999) 
32. Y.S. Myung, Mod. Phys. Lett. A 16, 2353 (2001)

33. A.M. Ghezelbash, R.B. Mann, J. High Energy Phys. 0201, 005 (2002)

34. V. Cardoso, J.P.S. Lemos, Phys. Rev. D 67, 084020 (2003)

35. S. Shankaranarayanan, Phys. Rev. D 67, 084026 (2003)

36. D. Kubizňák, F. Simovic. arXiv:1507.08630 [hep-th]

37. G. 't Hooft, Nucl. Phys. B 256, 727 (1985)

38. T. Jacobson, Phys. Rev. Lett. 75, 1260 (1995)
39. C. Rovelli, Phys. Rev. Lett. 77, 3288 (1996)

40. A. Strominger, C. Vafa, Phys. Lett. B 379, 99 (1996)

41. A. Ashtekar, J. Baez, A. Corichi, K. Krasnov, Phys. Rev. Lett. 80, 904 (1998)

42. W.A. Hiscock, Phys. Rev. D 31, 3288 (1985)

43. L. Herrera, N.O. Santos, A.F.F. Teixeira, A.Z. Wang, Class. Quantum Gravity 18, 3847 (2001) 